\title{
Diagnostic performance of a nomogram incorporating cribriform morphology for the prediction of adverse pathology in prostate cancer at radical prostatectomy
}

\author{
BAOJUN WANG $^{1 *}$, JIE GAO $^{1 *}$, QING ZHANG ${ }^{1 *}$, YAO FU ${ }^{2}$, GUANGXIANG LIU ${ }^{1}$, \\ CHENGWEI ZHANG ${ }^{1}$, WANG WEI ${ }^{1}$, HAIFENG HUANG ${ }^{1}$, JIONG SHI $^{2}$, DANYAN LI $^{3}$ and HONGQIAN GUO ${ }^{1}$ \\ Departments of ${ }^{1}$ Urology, ${ }^{2}$ Pathology and ${ }^{3}$ Radiology, Nanjing Drum Tower Hospital, The Affiliated \\ Hospital of Nanjing University Medical School, Nanjing, Jiangsu 210008, P.R. China
}

Received September 4, 2019; Accepted April 16, 2020

DOI: $10.3892 / \mathrm{ol} .2020 .11861$

\begin{abstract}
The aim of the present study was to develop a novel nomogram that incorporated clinical factors, imaging parameters and biopsy pathological factors (including cribriform morphology) to predict adverse pathology in prostate cancer (PCa). A total of 223 patients with $\mathrm{PCa}$, who had undergone preoperative multi-parametric magnetic resonance imaging and had a biopsy of Gleason pattern (GP) 4, absence of GP 5 and pure Grade Group (GG) 3 [Gleason score (GS) $3+4$, GS 4+3, GS 4+4], were retrospectively enrolled onto the study. The contribution of GG to the biopsy and Prostate Imaging Reporting and Data System (PI-RADS) score for PCa harboring adverse pathology were analyzed. Univariate and multivariate logistic regression analyses were performed to determine significant pathology predictors of adverse pathology for nomogram development. The nomogram was internally validated using bootstrapping with 1,000 iterations. The diagnostic performance of the nomogram was analyzed by receiver operating characteristics (ROC) analysis and decision curve analysis (DCA). A higher biopsy GG and PI-RADS score were associated with an increased likelihood of adverse pathology. Prostate specific antigen density (PSAD), biopsy GG, cribriform morphology on biopsy and PI-RADS score were significant predictors and were included in the nomogram. The ROC area under the curve of the nomogram was 0.88 (95\% confidence interval, 0.84-0.91), with a high specificity (0.91) and moderate sensitivity (0.72). The
\end{abstract}

Correspondence to: Dr Hongqian Guo, Department of Urology, Nanjing Drum Tower Hospital, The Affiliated Hospital of Nanjing University Medical School, 321 Zhongshan Road, Nanjing, Jiangsu 210008, P.R. China

E-mail:dr.ghq@nju.edu.cn

${ }^{*}$ Contributed equally

Key words: whole-mount pathological analysis, multi-parametric magnetic resonance imaging, cribriform morphology, nomogram, prostate cancer, adverse pathology novel nomogram was shown to have a higher net benefit for the prediction of adverse pathology in $\mathrm{PCa}$, compared with any individual factors determined by DCA. Overall, a novel nomogram incorporating PSAD, PI-RADS score, biopsy GG and cribriform morphology on biopsy was shown to perform well in the prediction of PCa harboring adverse pathology at the time of radical prostatectomy.

\section{Introduction}

Prostate cancer ( $\mathrm{PCa})$ is the most common type of cancer in males and the second most common cause of male cancer-associated mortality in Western countries (1). It is necessary to stage $\mathrm{PCa}$ accurately prior to surgery, to ensure appropriate treatment options, clinical decisions and patient counseling are applied. PCa with adverse pathology tends to be more aggressive and harbors poorer prognosis (1). Adverse pathology is defined as $\geq$ Grade Group 3 (GG 3)/Gleason score (GS) 4+3=7 radical prostatectomy (RP), extraprostatic extension (EPE), seminal vesicle invasion (SVI) or lymph node metastasis (LNM) (2). For patients with suspected EPE or SVI, surgical techniques may be modified to include, for example, a wider resection margin, enlarged lymph node dissection or nerve resection, in order to reduce the risk of positive surgical margin. In addition, for some patients with EPE or SVI, external beam radiation therapy may be a more appropriate choice compared with RP, which may have risks associated with incomplete resection (3). Multi-parametric magnetic resonance imaging (mpMRI) and MRI/ultrasound (US) fusion-targeted biopsy (TBx) are relatively new techniques for PCa detection. Studies have shown that TBx performs better in detecting clinically significant PCa (csPCa) compared with systematic biopsy (SB) alone $(2,4)$. Ahmed et al (5) found that mpMRI had a higher sensitivity compared with standard transrectal US (TRUS) in ruling out clinically insignificant diseases. Under prostate imaging reporting and data system (PI-RADS) version 2 (4), lesions receive an assessment PI-RADS score of 1-5, with higher scores being associated with a higher likelihood of clinically significant PCa (csPCa), which is when the cancer volume is $\geq 0.5 \mathrm{ml}$ and/or $\mathrm{GS} \geq 3+4$ and/or stage $\geq \mathrm{pT} 3$ (6). Certain studies have demonstrated that the GS of PCa biopsy 
specimens is strongly associated with adverse pathology at the time of RP, biochemical recurrence and poor prognosis, with higher GS being associated with a worse treatment response (5,7). Therefore, high-grade $\mathrm{PCa}$ is associated with a less favorable prognosis and impacts clinical decisions, such as determining the most appropriate treatment. Gleason pattern (GP) 4 is a heterogeneous group that is classified into several architectural patterns, including poorly formed, cribriform and fused glands, by the International Society of Urological Pathology (ISUP) (8). Compared with tumors with a non-cribriform pattern 4 (poorly-formed and fused glands), tumors with positive cribriform morphology are associated with a higher likelihood of EPE and metastasis $(9,10)$. Previous studies have suggested that cribriform morphology may be more aggressive and can optimize Gleason scoring $(6,11)$. In addition, studies have demonstrated that cribriform pattern in $\mathrm{PCa}$ is associated with an increased risk of biochemical recurrence and cancer-specific survival $(12,13)$.

Currently, several clinical nomograms have been developed, including GG and clinical factors, which can predict adverse pathology in PCa. However, to the best of our knowledge, no studies have incorporated pathological factors, such as cribriform morphology, into a nomogram for adverse pathology prediction. The aim of the present study was therefore to develop a novel nomogram incorporating biopsy cribriform morphology, imaging parameters and clinical factors to identify adverse pathology in PCa.

\section{Materials and methods}

Study population. A total of 657 patients who underwent RP at Nanjing Drum Tower Hospital (Nanjing, China), due to histologically-confirmed PCa, between August 2016 and March 2019 were retrospectively included in the present study. The following patients were excluded: i) Patients with histologically-confirmed PCa from other medical institutions $(n=169)$; ii) GP 5 on biopsy $(n=27)$; iii) pure GP 3 on biopsy $(\mathrm{n}=142)$; iv) patients with a main lesion of $<$ PI-RADS 3 on MRI ( $n=6)$; and v) patients who had undergone previous treatment, including hormone therapy $(n=82)$ and transurethral resection of the prostate $(n=8$; Fig. 1). Finally, 223 patients who had undergone preoperative mpMRI, had GP 4 and absence of GP 5 on biopsy specimens (GS 3+4, GS 4+3, GS $4+4)$ were included in the present study. The median age was 69 years (age range, 50-84 years). The median age of adverse pathology-negative group and adverse pathology-positive group were used to determine age grouping. The study was approved by The Ethics Committee of the Nanjing Drum Tower Hospital (approval no. 2017-147-01) and all patients provided informed and signed consent.

mpMRI examination and image evaluation. All patients underwent pelvic mpMRI using a 3.0-T MR scanner (Achieva 3.0 TTX; Philips Healthcare) using a 16-channel phased array coil, as previously described (14). Transverse/coronal/sagittal (18 slices; thickness, $3 \mathrm{~mm} / \mathrm{gap} 0.5 \mathrm{~mm}$; repetition time (TR), $3744 \mathrm{~ms}$; time of echo (TE), $120 \mathrm{~ms}$; number of signals acquired, 2; resolution, $1.49 \times 1.51 \mathrm{~mm}) \mathrm{T} 2$-weighted turbo spin-echo images were acquired. Diffusion weighted spin-echo echo-planar images (18 slices; thickness; $3 \mathrm{~mm}$; intersection gap, $1 \mathrm{~mm}$; TR, 925/TE, $41 \mathrm{~ms}$; number of signals acquired, 1; resolution, $3 \times 3 \mathrm{~mm}$; b-factor, 0/800/1500 sec/mm ${ }^{2}$ ) were acquired. T1 high-resolution isotropic volume with fat suppression, following a $30 \mathrm{ml}$ gadolinium injection was used for dynamic contrast-enhanced images (133 slices; thickness, $3 \mathrm{~mm}$; no intersection gap; TR, 3.1/TE, $1.46 \mathrm{~ms}$; number of signals acquired, 1 ; resolution, $1.49 \times 1.51 \mathrm{~mm}$; dynamic scan time, 00:06.9 min). All mpMRI imaging was reviewed by two radiologists with $>10$ years of experience with prostate mpMRI, who were aware of the PCa diagnosis for all cases but blinded to the final pathology, including the GS. Regions of interest (ROI), defined as regions with an abnormal signal on mpMRI, were contoured and scored using PI-RADS (6). The maximum length, width and height of the suspected lesion on apparent-diffusion coefficient sequence were measured, and the prostate volume was calculated using the following formula: $0.52 \mathrm{x}$ width $\mathrm{x}$ lenth $\mathrm{x}$ height'.

Biopsy protocol and pathological assessment. All biopsies were conducted using the mpMRI-US image registration system (Esaote ${ }^{\circledR}$ and RVS $^{\circledR}$ ) that provided real-time fusion of TRUS and mpMR images, in order to guide the biopsy needles transperineally. The biopsy started with TB, aiming at the center of suspicious lesions, using the free-hand transperineal (7) method by a senior urologist, and then standard systematic 12-core transperineal SB (blinded to the MRI target lesions) was conducted in all patients by another dedicated urologist. An 18-G automatic biopsy gun with a specimen size of $18 \mathrm{~mm}$ (Gallini Medical; www.gallinimedical.com) was used to obtain biopsy cores (14). Biopsy cores were graded by two genitourinary pathologists at Nanjing Drum Tower Hospital (Nanjing, China), in accordance with the ISUP guidelines (15). GG is based on the highest GG on TB and SB pathological outcomes. The GGs, used in parallel to the modified Gleason grading system, translate GSs in five distinct risk categories, where GG 1 is defined as GS 6, GG 2 as GS $3+4=7$, GG 3 as GS $4+3=7$, GG 4 as GS 8 and GG 5 as GS 9/10 (16). Cribriform morphology on biopsy was identified by genitourinary pathologists at Nanjing Drum Tower Hospital.

Whole-mount pathological evaluation. Following robotic-assisted RP, whole-mount tissues were fixed using $10 \%$ formalin, embedded in paraffin, microtome-cut into 4-5 mm slices and hematoxylin \& eosin-stained, according to the standard protocol (17). All whole mount histology slides were subsequently digitalized using a scanning system (NanoZoomer Digital Pathology System; Hamamatsu Photonics K.K.). All pathological images were interpreted by the genitourinary pathologists. To identify pathological ROI, tumor lesions were contoured, and corresponding GS were assigned. Cribriform morphology was identified by the genitourinary pathologists at Nanjing Drum Tower Hospital. For primary analyses, adverse pathology was defined as $\geq \mathrm{GG}$ 3/GS 4+3=7 RP, EPE, SVI or LNM (2).

Statistical analysis. All patient demographics, cribriform morphology on biopsy, RP pathological results and MRI findings were analyzed descriptively. Patients with or without adverse pathological characteristics, cribriform morphology 
on biopsy, biopsy GG and PI-RADS score were shown. Mann-Whitney $\mathrm{U}$ tests were used for continuous variables and the $\chi^{2}$ test for categorical variables. Univariate and multivariate logistic regression analyses were performed to determine significant predictors of adverse pathology for nomogram development. The performance of the novel nomogram in predicting adverse pathology was assessed by receiver operating characteristics (ROC) curves, area under the curve (AUC) values and 95\% confidence interval (CI), sensitivity and specificity were calculated. The extent of over- or underestimation of predicted probabilities relative to observed probabilities of adverse pathology was explored graphically using calibration plots, which were internally validated using bootstrapping with 1,000 iterations. Decision curve analysis (DCA) identified the optimal approach for adverse pathology detection by comparing the net benefit of each factor across different threshold probabilities. Statistical analyses were performed using $\mathrm{R}$ version 3.5.2 (8) (packages rms, pROC and ggplot 2) and R studio software [version 1.1.383; (9)]. DCA was performed using the DCA package (18). All descriptive analyses were performed using SPSS version 22.0 software (IBM Corp). For tests of all variables, $\mathrm{P}<0.05$ was considered to indicate a statistically significant difference.

\section{Results}

Patient characteristics. A total of 223 PCa patients, who underwent preoperative mpMRI, had GP 4 and absence of GP 5 (GS 3+4, GS 4+3, GS 4+4) in biopsy specimens, were retrospectively enrolled in the present study. Table I includes the characteristics of all enrolled patients with $\mathrm{PCa}$. The median age was 69 years, and the median PSA level was $13.16 \mathrm{ng} / \mathrm{ml}$.

Characteristics of patients with or without adverse pathology. The demographics, biopsy GG, PI-RADS score and cribriform architecture on biopsy with and without adverse pathology are shown in Table II. The patients harboring adverse pathology were older compared with those who did not (71.00 vs. 68.00 years; $\mathrm{P}=0.04$ ). The PSA level of patients with adverse pathology was higher compared with patients without (14.59 vs. $10.20 \mathrm{ng} / \mathrm{ml} ; \mathrm{P}<0.01)$. Compared with patients without adverse pathology, the maximum lesion diameter was longer in the MRI of patients with adverse pathology $(1.90$ vs. $1.20 \mathrm{~cm}, \mathrm{P}<0.01)$. The contribution of PI-RADS score and GG on biopsy was also observed. For PI-RADS score $3 \mathrm{PCa}$, the detection rates of adverse pathology were 7/23 (30.4\%). In contrast, PI-RADS score 4 PCa was more likely to have adverse pathology [41/70 (58.6\%) vs. 7/23 (30.4\%) $\mathrm{P}=0.02$ ]. However, the detection rates of adverse pathology for PI-RADS score $5 \mathrm{PCa}$ were the highest [110/130 (84.6\%) vs. $7 / 23$ (30.4\%); $\mathrm{P}<0.01]$. The present study demonstrated that a higher preoperative GG on biopsy was associated with a higher likelihood of adverse pathology in PCa. The prediction rates of biopsy GG 2, GG 3, GG 4 were 24/59 (40.7\%), 57/79 (72.2\%) and 77/85 (90.6\%), respectively. For cribriform morphology-positive $\mathrm{PCa}$, the rates of adverse pathology detection were 60/68 (88.2\%). By contrast, cribriform morphology-negative PCa was associated with a decreased likelihood of adverse pathology [98/155 (63.2\%) vs. $60 / 68$ (88.2\%); $\mathrm{P}<0.01]$. Representative radiopathological
Table I. Characteristics of all 223 patients with prostate cancer included in the present study.

\begin{tabular}{lc}
\hline Characteristics & Value \\
\hline Age, years (range) & $69(50-84)$ \\
PSA level, ng/ml (range) & $13.16(4.02-110.48)$ \\
Prostate volume, ml (range) & $30.1(8.20-119.00)$ \\
PSAD, ng/ml (range) & $0.45(0.05-3.44)$ \\
Maximum diameter on & $1.70(0.20-4.60)$ \\
MRI, cm (range) & \\
mpMRI findings, n (\%) & \\
PI-RADS score 3 & $23(10.3)$ \\
PI-RADS score 4 & $70(31.4)$ \\
PI-RADS score 5 & $130(58.3)$ \\
Suspected extraprostatic extension & $113(50.7)$ \\
Suspected seminal vesicle invasion & $20(9.0)$ \\
Suspected lymph node invasion & $6(2.7)$ \\
Grade Group on RP, n (\%) & \\
1 & $2(0.9)$ \\
2 & $97(43.5)$ \\
3 & $89(39.9)$ \\
4 & $17(7.6)$ \\
5 & $18(8.1)$ \\
pT stage, n (\%) & \\
2 & $93(41.7)$ \\
$3 \mathrm{a}$ & $97(43.5)$ \\
3b & $31(13.9)$ \\
4 & $2(0.9)$ \\
pN stage, n (\%) & \\
0 & $208(0.93)$ \\
Cribriform morphology on biopsy, n $(\%)$ & $15(0.07)$ \\
Negative & $155(69.5)$ \\
Positive & $68(30.5)$ \\
&
\end{tabular}

PSA, prostate specific antigen; PSAD, prostate specific antigen density; Maximum diameter, maximum diameter of lesion on MRI; $\mathrm{RP}$, radical prostatectomy; mpMRI, multiparametric magnetic resonance imaging; PI-RADS, Prostate Imaging Reporting and Data System; pN, pathological N stage; pT, pathological T stage.

matching of cribriform morphology-positive lesion on biopsy and RP are shown in Fig. 2.

Univariate and multivariate logistic regression analyses for the detection of adverse pathology. In univariate logistic regression analysis for the prediction of adverse pathology in $\mathrm{PCa}$, prostate specific antigen density (PSAD) $(\mathrm{P}<0.01)$, maximum lesion diameter on MRI $(\mathrm{P}<0.01)$, cribriform morphology on biopsy $(\mathrm{P}<0.01)$, biopsy GG $(\mathrm{P}<0.01)$ and PI-RADS score $(\mathrm{P}<0.01)$ were significant factors. In multivariate logistic regression analysis for the prediction of adverse pathology, PSAD $(\mathrm{P}=0.03)$, cribriform morphology $(\mathrm{P}=0.02)$, biopsy $\mathrm{GG}(\mathrm{P}<0.01)$ and $\mathrm{PI}-\mathrm{RADS}$ score $(\mathrm{P}<0.01)$ were significant factors and were 
Table II. Characteristics of patients with or without adverse pathology in the study ( $\mathrm{n}=158$ and 64 , respectively).

\begin{tabular}{lcc}
\hline & \multicolumn{2}{c}{ Adverse pathology } \\
\cline { 2 - 3 } Characteristics & Negative & Positive \\
\hline Age, years (range) & $68(52-81)$ & $71(50-84)$ \\
PSA level, ng/ml (range) & $10.20(4.02-100)$ & $14.59(4.00-110.48)$ \\
Prostate volume, ml (range) & $27.9(15.8-85.90)$ & $33.2(8.20-119.00)$ \\
PSAD, ng/ml (range) & $0.34(0.05-2.83)$ & $0.49(0.05-3.44)$ \\
Maximum diameter on MRI, cm (range) & $1.20(0.20-3.80)$ & $1.90(0.50-4.60)$ \\
PI-RADS score, n (\%) & & $7(30.4)$ \\
3 & $16(69.6)$ & $41(58.6)$ \\
4 & $29(41.4)$ & $110(84.6)$ \\
5 & $20(15.4)$ & $24(40.7)$ \\
Grade Group on biopsy, $\mathrm{n}(\%)$ & & $57(72.2)$ \\
2 & $35(59.3)$ & $77(90.6)$ \\
3 & $22(27.8)$ & $<0.017$ \\
4 & $8(9.4)$ & $<0.01$ \\
Cribriform morphology on biopsy, $\mathrm{n}(\%)$ & & $60.02^{\mathrm{a}}$ \\
Negative & $57(36.8)$ & $60(88.2)$ \\
\hline
\end{tabular}

PSA, prostate specific antigen; PSAD, prostate specific antigen density; Maximum diameter, maximum diameter of lesion on MRI; PI-RADS,

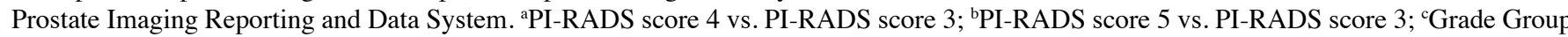
3 vs. Grade Group 2; ${ }^{\mathrm{d}}$ Grade Group 4 vs. Grade Group 2; ${ }^{\mathrm{e}}$ Cribriform morphology positive vs. negative.

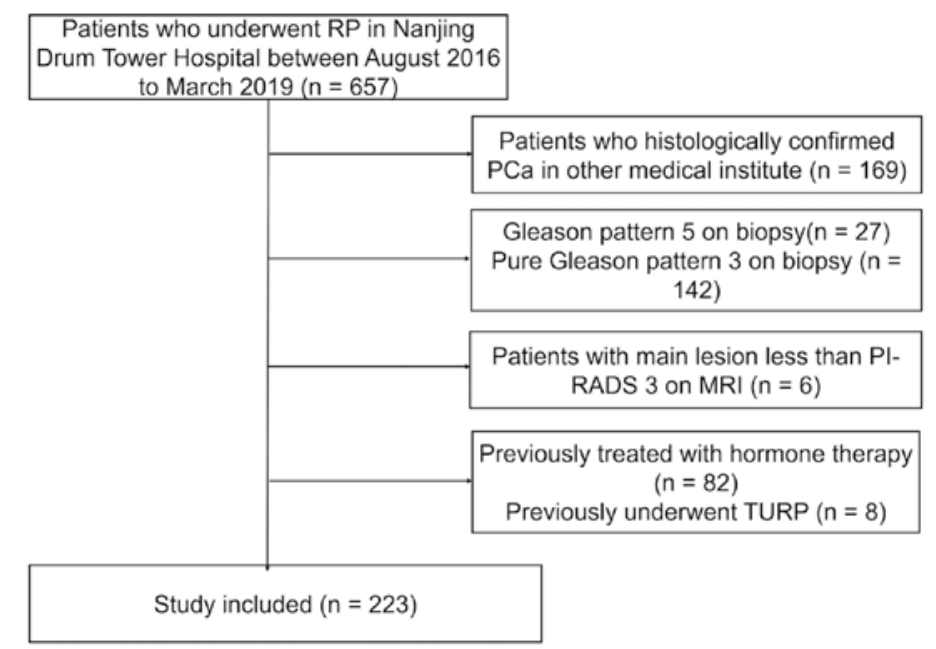

Figure 1. Flowchart detailing the exclusion criteria of the present study. RP, radical prostatectomy; PCa, prostate cancer; PI-RADS, Prostate Imaging Reporting and Data System; TURP, transurethral resection of the prostate.

included in the nomogram construction (Table III; Fig. 3). The AUC of the novel nomogram was 0.88 (95\% CI, 0.84-0.91), with a high specificity (0.91) and moderate sensitivity $(0.72$; Fig. 4). Bootstrapped calibration plots of the nomogram (Fig. 5) demonstrated that there were no significant deviations of the predicted risk from the observed risk of adverse pathology in PCa over the entire range (mean absolute error=0.017). In DCA, compared with PSAD, cribriform morphology on biopsy, biopsy GG, PI-RADS score, the nomogram had a higher net benefit for the prediction of adverse pathology (Fig. 6).

\section{Discussion}

In the present study, a novel nomogram incorporating PSAD, PI-RADS score, cribriform morphology on biopsy and biopsy GG was developed, and showed a good prediction performance for PCa harboring adverse pathology.

Prediction models that combine clinical stage, serum PSA level and Gleason grade in biopsy specimens are commonly used in clinical practice to predict the pathological stage of PCa. The Partin (19) and Memorial Sloan Kettering Cancer 

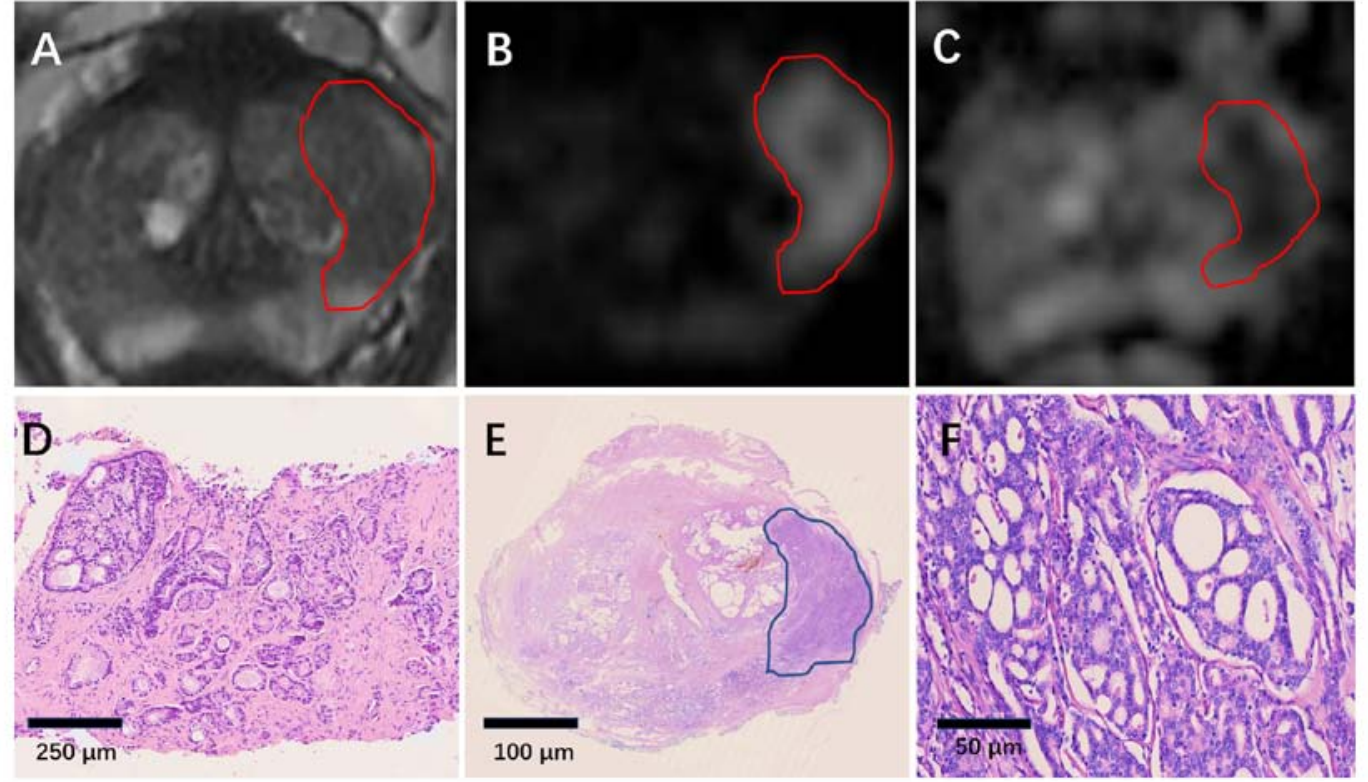

Figure 2. A representative case of prostate cancer harboring adverse pathology. A 67-year-old man with protein specific antigen density of 9.9 ng/ml, prostate volume of $23.90 \mathrm{ml}$. (A) T2-weighted image shows a significantly hypointense lesion in the left peripheral zone (red). (B) Diffusion weighted imaging with b1500 shows a high signal on the left peripheral zone (red). (C) Lesion was hypointense on apparent-diffusion coefficient map (red), MRI/ultrasound fusion-guided biopsy and systematic biopsy was conducted and showed the highest Gleason score 3+4 (Grade Group 2). All the finding results in a Prostate Imaging Reporting and Data System score 5. (D) MRI/ultrasound fusion-guided biopsy and systematic biopsy was conducted and showed Gleason score 3+4 with cribriform morphology. The magnification of the picture is at $\mathrm{x} 250$ magnification. (E) Whole mount histology demonstrated that the lesion was a clinically significant with Gleason score 4+3=7, with extraprostatic extension. Magnification, x100. (F) Representative cribriform morphology derived from whole mount histology. The magnification of the picture is $50 \mu \mathrm{m}$.

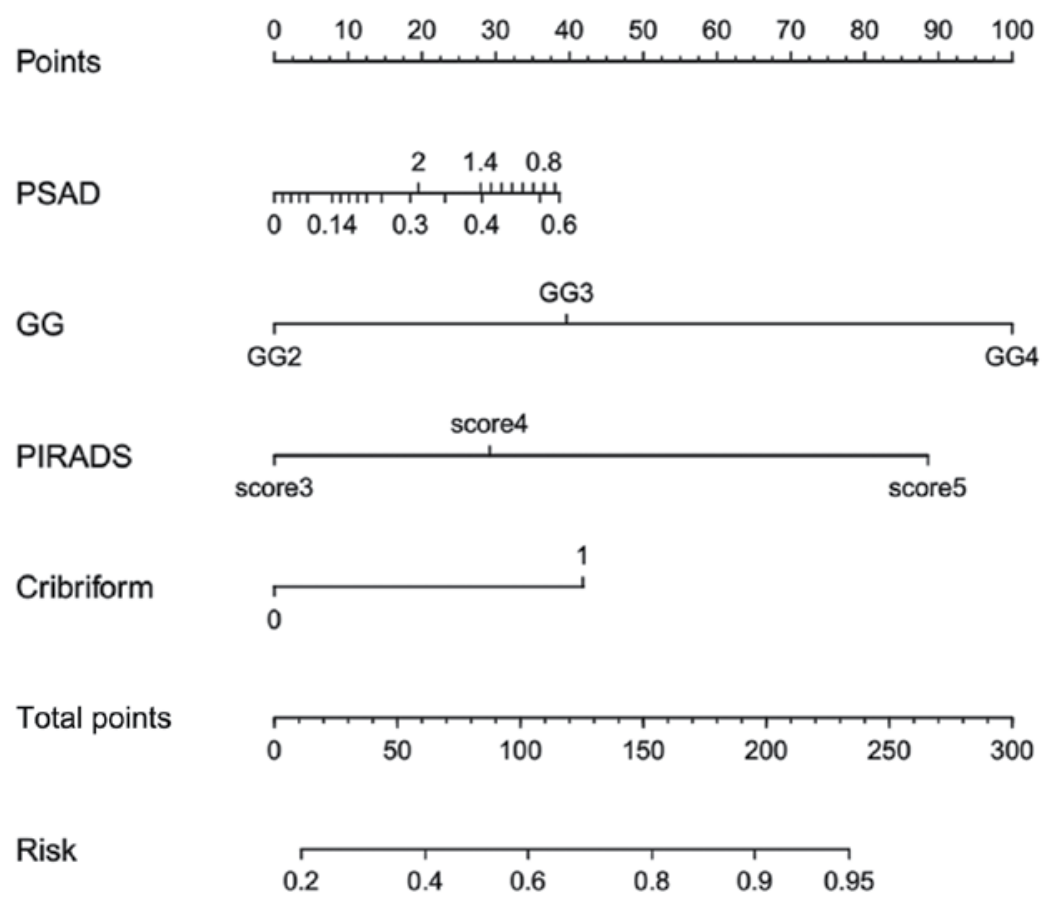

Figure 3. Nomogram to predict adverse pathology in prostate cancer including PI-RADS score, PSAD, cribriform morphology on biopsy and biopsy GG. PI-RADS, Prostate Imaging Reporting and Data System; PSAD, protein specific antigen density; GG, grade group.

Center pre-RP (20) nomograms are examples of predicted models widely used for preoperative decision-making. However, imaging parameters and pathological factors, such as mpMRI and cribriform pattern, are not included in the nomogram, which are generally recognized as valuable factors for improved detection of PCa harboring adverse pathology. Rayn et al (21) showed that MRI, alone or combined with standard clinical nomograms, provides additional predictive value of adverse pathology at the time of RP. Although MRI was incorporated, the Rayn et al nomogram had certain 
Table III. Univariate and multivariate logistic regression analyses for the detection of adverse pathology in prostate cancer.

\begin{tabular}{|c|c|c|c|c|}
\hline \multirow[b]{2}{*}{ Variable and intercept } & \multicolumn{2}{|c|}{ Univariate logistic regression } & \multicolumn{2}{|c|}{ Multivariate logistic regression } \\
\hline & OR $(95 \% \mathrm{CI})$ & P-value & OR $(95 \% \mathrm{CI})$ & P-value \\
\hline Age, per 5 years & $1.09(0.94-1.26)$ & 0.24 & NA & NA \\
\hline PSAD, interquartile OR & $2.26(1.81-2.81)$ & $<0.01$ & $1.40(1.03-1.92)$ & 0.03 \\
\hline Maximum diameter on MRI, interquartile OR & $2.30(1.84-2.87)$ & $<0.01$ & $0.85(0.54-1.32)$ & 0.49 \\
\hline \multicolumn{5}{|l|}{ Cribriform morphology on biopsy } \\
\hline No & 1 (Ref) & & 1 (Ref) & \\
\hline Yes & $20.70(6.34-67.55)$ & $<0.01$ & $4.45(1.24-15.90)$ & 0.02 \\
\hline \multicolumn{5}{|l|}{ Grade Group on biopsy } \\
\hline 2 & 1 (Ref) & & 1 (Ref) & \\
\hline 3 & $3.14(1.60-6.17)$ & $<0.01$ & $2.05(0.97-4.36)$ & $<0.01$ \\
\hline 4 & $9.00(4.67-17.35)$ & $<0.01$ & $4.39(2.11-9.17)$ & $<0.01$ \\
\hline \multicolumn{5}{|l|}{ PI-RADS score } \\
\hline 3 & 1 (Ref) & & 1 (Ref) & \\
\hline 4 & $4.53(2.12-9.69)$ & $<0.01$ & $2.99(1.27-7.07)$ & $<0.01$ \\
\hline 5 & $21.77(10.03-47.24)$ & $<0.01$ & $8.48(2.47-29.14)$ & $<0.01$ \\
\hline
\end{tabular}

PSAD, prostate specific antigen density; Maximum diameter, maximum diameter of lesion on MRI; OR, odds ratio; CI, confidence interval; PI-RADS, Prostate Imaging Reporting and Data System; NA, not applicable; Ref, reference.

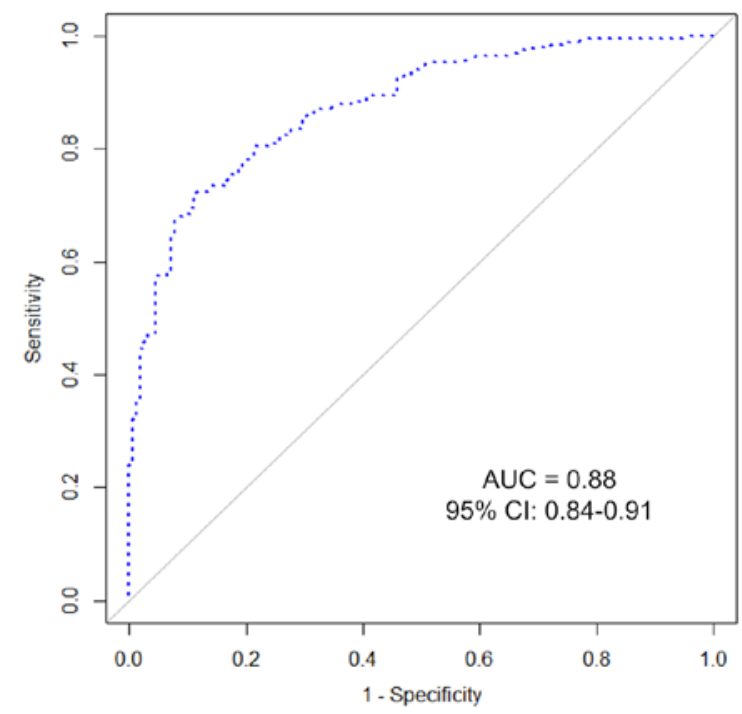

Figure 4. Receiver operating characteristic curve analysis for the performance of adverse pathology nomogram. AUC, area under the curve; CI, confidence interval.

limitations. First, PI-RADS version 2 (v2) was not used to evaluate the effect of MRI uniformly. PI-RADS v2 is widely accepted by peer experts for MRI accessing and clinical practice as guidance (6). It was observed in the present study that a higher PI-RADS score was associated with an increased likelihood of adverse pathology. Junker et al (22), reported that PI-RADS 4 and PI-RADS 5 were associated with high-grade PCa. Lim et al (23), showed that PI-RADS 5 was associated with a higher GSs and EPE compared with
PI-RADS 4. Secondly, pathological factors were not included in the nomogram of Rayn et al. The present study incorporated pathological factors, including cribriform morphology on biopsy, into the nomogram, and observed a favorable prediction performance for adverse pathology.

Sarbay et al (24), reported that cribriform morphology on prostate biopsy was associated with an increased likelihood of positive surgical margins and extraprostatic extension at the time of RP. Another study showed that perineural invasion on biopsy was associated with adverse pathology at the time of RP; however, perineural invasion was inferior to cribriform pattern in predicting non-organ-confined disease at the time of RP (25). Over the past few years, several studies have shown that cribriform-positive PCa is associated with an increased likelihood of lymph node invasion and distant metastasis $(10,26)$. The present study also demonstrated that the presence of cribriform morphology on biopsy was associated with an increased risk of adverse pathology in PCa compared with RP pathological outcomes. Therefore, cribriform morphology on biopsy, which was incorporated into the present nomogram, is a significant factor for predicting $\mathrm{PCa}$ harboring adverse pathology. Although not yet practiced clinically, and given that cribriform morphology has not been extensively studied, reporting cribriform morphology on prostate biopsies is strongly encouraged by some experts. Suggestions for accommodating the presence of cribriform cancer into the 2014 GG scheme have been made (6). The present team has recently been trying to encourage pathologists to report cribriform morphology on prostate biopsies at Nanjing Drum Tower Hospital. In addition, a higher GG was found to be associated with an increased risk of adverse pathology in PCa compared with RP pathological results. Aminsharifi et al (27), showed that a higher preoperative biopsy GG is linked to a higher 


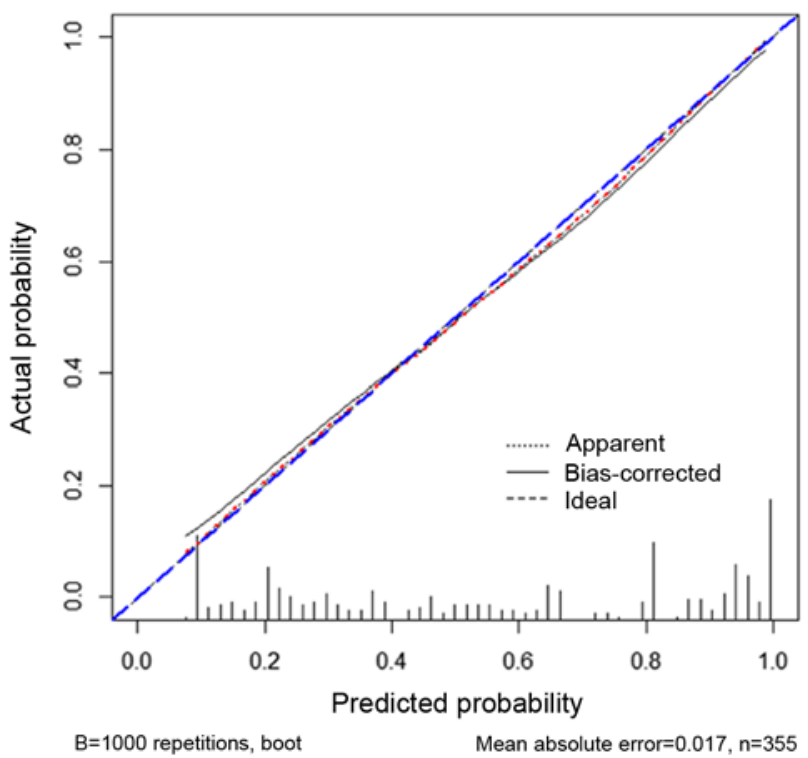

Figure 5. Calibration plots for the nomogram to predict adverse pathology in prostate cancer.

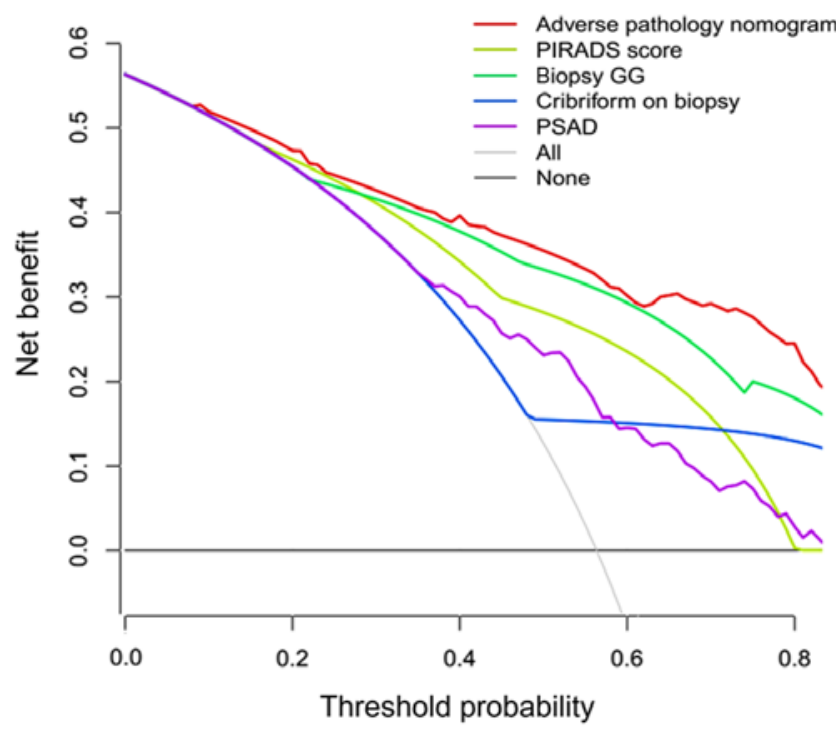

Figure 6. Net decision curve analysis demonstrating the benefit of adverse pathology nomogram for prediction of adverse pathology in prostate cancer. PI-RADS, Prostate Imaging Reporting and Data System; GG, grade group; PSAD, protein specific antigen density.

risk of adverse histopathological findings at the time of RP. Hence, for a patient with a high biopsy GG and positive cribriform morphology on biopsy, a careful evaluation should be conducted to ensure suitable treatment strategies are followed.

A strength of the present study was that cribriform morphology on prostate biopsy was incorporated into the nomogram. To the best of our knowledge, the present study is the first to incorporate pathological factors into the nomogram predicting adverse pathology in PCa. In addition, the final pathological results were based on whole-mount pathological analyses. However, the present study had certain limitations. Firstly, it was a retrospective study and therefore has the bias associated with this type of study. Secondly, only a limited number of patients fitted the inclusion criteria.
Thirdly, the final pathology was used as a reference standard, thus a selection bias is likely to have occurred, in that patients with lower-risk disease are less likely to have RP and negative- or low-risk patients were not included. Nonetheless, the final RP specimen is the most accurate reference standard to determine the presence or absence of PCa harboring adverse pathology. Fourthly, according to the study aim, only patients with a GP of 4 and without pure GP 3 and GP 5 were enrolled in the study, which might restrict clinical application to the general population. However, Stroup et al (28) showed that any GP 5 on biopsy, which indicated a poor prognosis, is associated with a higher likelihood of metastasis and PCa-specific mortality and adverse outcomes. In addition, an increasing number of studies have demonstrated that pure GP 3 diseases on biopsy have negligible PCa-specific mortality (29) and metastasis (30). Therefore, the inclusion criteria for the present study were considered suitable and in line with clinical practice. Finally, because of short postoperative time, follow-up of patients to evaluate the predictive value of the nomogram in biochemical recurrence was not conducted. Therefore, future studies should be conducted to verify the results of the present imaging models.

The present study found that a novel nomogram incorporating PSAD, PI-RADS score, cribriform morphology on biopsy and biopsy GG provided a significant predictive ability for PCa harboring adverse pathology at the time of RP. Urologists can use this nomogram to counsel patients regarding surgery techniques and future therapies and help them make significant management decisions. In future, the present nomogram should be validated in an independent cohort.

\section{Acknowledgements}

The authors of the present study would like to thank Dr Guo at the Department of Urology, Nanjing Drum Tower Hospital, The Affiliated Hospital of Nanjing University Medical School (Nanjing, China) for his help in project development, analyzing the data and editing the manuscript.

\section{Funding}

This research was supported by The National Natural Science Foundation of China (grant nos. 81772710 and 81572519), The Project of Invigorating Health Care through Science, Technology and Education, Jiangsu Provincial Key Medical Discipline (grant no. ZDXKB2016014), The National Natural Science Foundation of China (grant no. 81802535), China Postdoctoral Fund (grant no. 223427) and The Nanjing Medical Science and Technique Development Foundation (grant no. YKK 18064).

\section{Availability of data and materials}

The datasets used and/or analyzed during the current study are available from the corresponding author on reasonable request.

\section{Authors' contributions}

BW, JG and QZ conceived the study and contributed equally to data collection, data analysis, manuscript writing and 
editing. DL performed radiological analysis. CZ and GL performed clinical analyses associated with introducing the concept of csPCa, the Gleason group, hormone therapy and prostate biopsy. WW and HH performed MRI/ultrasound fusion-targeted biopsy and systematic biopsy. YF and JS performed pathological analysis. HG was involved in project development, data analysis and edited the manuscript. All authors read and approved the final manuscript.

\section{Ethics approval and consent to participate}

The present study was approved by The Ethics Committee of the Nanjing Drum Tower Hospital (approval no. 2017-147-01) and all patients provided informed and signed consent.

\section{Patient consent for publication}

Consent for publication was been obtained for each participant according to federal and institutional guidelines $(14,31)$.

\section{Competing interests}

The authors declare that they have no competing interests.

\section{References}

1. Center MM, Jemal A, Lortet-Tieulent J, Ward E, Ferlay J, Brawley $\mathrm{O}$ and Bray $\mathrm{F}$ : International variation in prostate cancer incidence and mortality rates. Eur Urol 61: 1079-1092, 2012.

2. Dean LW, Assel M, Sjoberg DD, Vickers AJ, Al-Ahmadie HA, Chen YB, Gopalan A, Sirintrapun SJ, Tickoo SK, Eastham JA, et al: Clinical usefulness of total length of Gleason pattern 4 on biopsy in men with grade group 2 prostate cancer. J Urol 201: 77-82, 2019.

3. Boehmer D, Maingon P, Poortmans P, Baron MH, Miralbell R, Remouchamps V, Scrase C, Bossi A and Bolla M; EORTC radiation oncology group: Guidelines for primary radiotherapy of patients with prostate cancer. Radiother Oncol 79: 259-269, 2006.

4. Siddiqui MM, Rais-Bahrami S, Turkbey B, George AK, Rothwax J, Shakir N, Okoro C, Raskolnikov D, Parnes HL, Linehan WM, et al: Comparison of MR/ultrasound fusion-guided biopsy with ultrasound-guided biopsy for the diagnosis of prostate cancer. JAMA 313: 390-397, 2015.

5. Ahmed HU, El-Shater Bosaily A, Brown LC, Gabe R, Kaplan R, Parmar MK, Collaco-Moraes Y, Ward K, Hindley RG, Freeman A, et al: Diagnostic accuracy of multi-parametric MRI and TRUS biopsy in prostate cancer (PROMIS): A paired validating confirmatory study. Lancet 389: 815-822, 2017.

6. Weinreb JC, Barentsz JO, Choyke PL, Cornud F, Haider MA, Macura KJ, Margolis D, Schnall MD, Shtern F, Tempany CM, et al: PI-RADS prostate imaging-reporting and data system: 2015, version 2. Eur Urol 69: 16-40, 2016.

7. Zhou P, Chen MH, McLeod D, Carroll PR, Moul JW and D'Amico AV: Predictors of prostate cancer-specific mortality after radical prostatectomy or radiation therapy. J Clin Oncol 23: 6992-6998, 2005.

8. Epstein JI, Allsbrook WC Jr, Amin MB, Egevad LL and Committee IG; ISUP Grading Committee: The 2005 international society of urological pathology (ISUP) consensus conference on Gleason grading of prostatic carcinoma. Am J Surg Pathol 29: 1228-1242, 2005.

9. Kweldam CF, Wildhagen MF, Steyerberg EW, Bangma CH, van der Kwast TH and van Leenders GJ: Cribriform growth is highly predictive for postoperative metastasis and disease-specific death in Gleason score 7 prostate cancer. Mod Pathol 28: 457-464, 2015.

10. Siadat F, Sykes J, Zlotta AR, Aldaoud N, Egawa S, Pushkar D Kuk C, Bristow RG, Montironi R and van der Kwast T: Not all Gleason pattern 4 prostate cancers are created equal: A study of latent prostatic carcinomas in a cystoprostatectomy and autopsy series. Prostate 75: 1277-1284, 2015.
11. McKenney JK, Wei W, Hawley S, Auman H, Newcomb LF, Boyer HD, Fazli L, Simko J, Hurtado-Coll A, Troyer DA, et al: Histologic grading of prostatic adenocarcinoma can be further optimized: Analysis of the relative prognostic strength of individual architectural patterns in 1275 patients from the canary retrospective Cohort. Am J Surg Pathol 40: 1439-1456, 2016.

12. Kir G, Sarbay BC, Gümüş E and Topal CS: The association of the cribriform pattern with outcome for prostatic adenocarcinomas. Pathol Res Pract 210: 640-644, 2014.

13. Harding-Jackson N, Kryvenko ON, Whittington EE, Eastwood DC, Tjionas GA, Jorda $\mathrm{M}$ and Iczkowski KA: Outcome of Gleason $3+5=8$ prostate cancer diagnosed on needle biopsy: Prognostic comparison with Gleason 4+4=8. J Urol 196: 1076-1081, 2016.

14. Zhang Q, Wang W, Zhang B, Shi J, Fu Y, Li D, Guo S, Zhang S, Huang $\mathrm{H}$, Jiang $\mathrm{X}$, et al: Comparison of free-hand transperineal mpMRI/TRUS fusion-guided biopsy with transperineal 12-core systematic biopsy for the diagnosis of prostate cancer: A single-center prospective study in China. Int Urol Nephrol 49: 439-448, 2017.

15. Epstein JI, Egevad L, Amin MB, Delahunt B, Srigley JR and Humphrey PA; Grading Committee: The 2014 international society of urological pathology (ISUP) consensus conference on Gleason grading of prostatic carcinoma: Definition of grading patterns and proposal for a new grading system. Am J Surg Pathol 40: 244-252, 2016.

16. Magi-Galluzzi C, Montironi R and Epstein JI: Contemporary Gleason grading and novel grade groups in clinical practice. Curr Opin Urol 26: 488-492, 2016.

17. McNeal JE and Haillot O: Patterns of spread of adenocarcinoma in the prostate as related to cancer volume. Prostate 49: 48-57, 2001.

18. Vickers AJ, Cronin AM, Elkin EB and Gonen M: Extensions to decision curve analysis, a novel method for evaluating diagnostic tests, prediction models and molecular markers. BMC Med Inform Decis Mak 8: 53, 2008.

19. Huang Y, Isharwal S, Haese A, Chun FK, Makarov DV, Feng Z, Han M, Humphreys E, Epstein JI, Partin AW and Veltri RW: Prediction of patient-specific risk and percentile cohort risk of pathological stage outcome using continuous prostate-specific antigen measurement, clinical stage and biopsy Gleason score. BJU Int 107: 1562-1569, 2011.

20. Ohori M, Kattan MW, Koh H, Maru N, Slawin KM, Shariat S, Muramoto M, Reuter VE, Wheeler TM and Scardino PT: Predicting the presence and side of extracapsular extension: A nomogram for staging prostate cancer. J Urol 171: 1844-1849, 2004.

21. Rayn KN, Bloom JB, Gold SA, Hale GR, Baiocco JA, Mehralivand S, Czarniecki M, Sabarwal VK, Valera V, Wood BJ, et al: Added value of multiparametric magnetic resonance imaging to clinical nomograms for predicting adverse pathology in prostate cancer. J Urol 200: 1041-1047, 2018.

22. Junker D, Quentin M, Nagele U, Edlinger M, Richenberg J, Schaefer G, Ladurner M, Jaschke W, Horninger W and Aigner F: Evaluation of the PI-RADS scoring system for mpMRI of the prostate: A whole-mount step-section analysis. World J Urol 33: 1023-1030, 2015.

23. Lim CS, McInnes MDF, Lim RS, Breau RH, Flood TA, Krishna S, Morash C, Shabana WM and Schieda N: Prognostic value of prostate imaging and data reporting system (PI-RADS) v. 2 assessment categories 4 and 5 compared to histopathological outcomes after radical prostatectomy. J Magn Reson Imaging 46: 257-266, 2017.

24. Sarbay BC, Kir G, Topal CS and Gumus E: Significance of the cribriform pattern in prostatic adenocarcinomas. Pathol Res Pract 210: 554-557, 2014.

25. Flood TA, Schieda N, Keefe DT, Morash C, Bateman J, Mai KT, Belanger EC, Robertson SJ and Breau RH: Perineural invasion on biopsy is associated with upstaging at radical prostatectomy in Gleason score 3+4=7 prostate cancer. Pathol Int 66: 629-632, 2016

26. Dong F, Yang P, Wang C, Wu S, Xiao Y, McDougal WS, Young RH and Wu CL: Architectural heterogeneity and cribriform pattern predict adverse clinical outcome for Gleason grade 4 prostatic adenocarcinoma. Am J Surg Pathol 37: 1855-1861, 2013.

27. Aminsharifi A, Schulman A, Howard LE, Tay KJ, Amling CL, Aronson WJ, Cooperberg MR, Kane CJ, Terris MK, Freedland SJ and Polascik TJ: Influence of African American race on the association between preoperative biopsy grade group and adverse histopathologic features of radical prostatectomy. Cancer 125: 3025-3032, 2019. 
28. Stroup SP, Moreira DM, Chen Z, Howard L, Berger JH, Terris MK, Aronson WJ, Cooperberg MR, Amling CL, Kane CJ and Freedland SJ: Biopsy detected Gleason pattern 5 is associated with recurrence, metastasis and mortality in a cohort of men with high risk prostate cancer. J Urol 198: 1309-1315, 2017.

29. Eggener SE, Scardino PT, Walsh PC, Han M, Partin AW, Trock BJ, Feng Z, Wood DP, Eastham JA, Yossepowitch O, et al: Predicting 15-year prostate cancer specific mortality after radical prostatectomy. J Urol 185: 869-875, 2011.

30. Liu JJ, Lichtensztajn DY, Gomez SL, Sieh W, Chung BI, Cheng I and Brooks JD: Nationwide prevalence of lymph node metastases in Gleason score 3+3=6 prostate cancer. Pathology 46: 306-310, 2014.
31. Gao J, Zhang C, Zhang Q, Fu Y, Zhao X, Chen M, Zhang B, Li D, Shi J, Wang F and Guo H: Diagnostic performance of ${ }^{68} \mathrm{Ga}$-PSMA PET/CT for identification of aggressive cribriform morphology in prostate cancer with whole-mount sections. Eur J Nucl Med Mol Imaging 46: 1531-1541, 2019.

(i) (3) This work is licensed under a Creative Commons Attribution-NonCommercial-NoDerivatives 4.0 International (CC BY-NC-ND 4.0) License. 